\title{
LOS ACUERDOS REGIONALES DE INTEGRACIÓN, UNA ALTERNATIVA DE DESARROLLO*
}

\author{
Manuela TAMAYO USME**
}

Presentado: 12 de junio de 2015 - Aprobado: 21 de septiembre de 2015

\section{Resumen}

A partir de la Segunda Guerra Mundial las relaciones jurídicas entre los diferentes países que integran la comunidad internacional sufrieron grandes transformaciones derivadas, fundamentalmente, del nuevo orden de poder que surgió de la conflagración universal que se dio entre los años 1938 y 1945. Esta nueva organización parte de las relaciones comerciales que hacen necesaria la integración entre los diferentes Estados, a fin de alcanzar niveles de desarrollo sostenibles en un mundo cada vez más especializado en la cadena de producción. Esta situación va a llevar a que, en las distintas subregiones del planeta, surjan Acuerdos Regionales de Integración que van a pretender unir los esfuerzos de los Estados, en procura de alcanzar una capacidad económica global que permita un mayor desarrollo. A esta tendencia no se escapa América Latina, a pesar de las dificultades que se presentan debido al resquebrajamiento ideológico de los distintos

* Artículo derivado de la investigación denominada "Acuerdos Regionales de Integración. Derecho latinoamericano, presente y futuro", adscrita al semillero de investigaciones sociohumanísticas de la Facultad de Derecho de la Fundación Universitaria Autónoma de las Américas.

** Estudiante de Derecho en la Facultad de Derecho de la Fundación Universitaria Autónoma de las Américas. Correo electrónico: tamayousme@ hotmail.com 
grupos que componen la región y a los intereses particulares de los gremios políticos, industriales y comerciales que ven, en la atomización de los Estados, una manera particular de alcanzar sus propios intereses, todo lo cual ha llevado al fracaso los intentos por alcanzar una verdadera integración regional.

Palabras clave: Acuerdo Regional de Integración, Aranceles, Ideología, Regionalización.

\title{
REGIONAL INTEGRATION AGREEMENTS, A DEVELOPMENT ALTERNATIVE
}

\begin{abstract}
Since World War II the legal relationships between the different countries of the international community suffered great transformations derived fundamentally of the new order of power that came from the universal conflagration that occurred between 1938 and 1945. This new organization part of the business relationships that requires the integration between the different States in order to achieve sustainable levels of development in an increasingly specialized world. This situation will lead to, in the different sub-regions of the planet, Regional Agreements of integration that will unite the efforts of States, seeking to reach a global economic capacity to allow further development arise. This trend in Latin America is not the exception despite the difficulties that arise due to the ideological breakdown of the various groups that make up the region and the interests of politicians, industry and trade unions that are in the atomization of the states, a particular way to achieve their own interests, all of which has led to the failure of attempts to achieve true regional integration.
\end{abstract}

Keywords: Regional Integration Agreement, tariffs, ideology, regionalization. 


\section{ACCORDS D'INTEGRATION REGIONALE, UNE ALTERNATIVE DE DEVELOPPEMENT}

\section{Résumé}

Depuis la Seconde Guerre mondiale, les relations juridiques entre les différents pays de la communauté internationale ont subi de grandes transformations dérivées fondamentalement du nouvel ordre de pouvoir qui est venu de la conflagration universelle qui a eu lieu entre 1938 et 1945. Cette nouvelle organisation part des relations d'affaires qui nécessitent l'intégration entre les différents Etats afin de parvenir à des niveaux durables de développement dans un monde plus spécialisé dans la chaîne de production. Cette situation va conduire à que dans les différentes sous-régions de la planète, les accords régionaux d'intégration qui vont unir les efforts des Etats, en cherchant à atteindre une capacité économique mondiale pour permettre la poursuite du développement. Cette tendance en Amérique latine ne fait pas exception, malgré les difficultés qui se posent en raison de la rupture idéologique des différents groupes qui composent la région et les intérêts des politiciens, l'industrie et les syndicats qui sont dans l'atomisation des États- une façon particulière d'atteindre leurs propres intérêts, tout ce qui a conduit à l'échec des tentatives pour parvenir à une véritable intégration régionale.

Mots-clés: Accord de l'intégration régionale, les droits de douane, l'idéologie, la régionalisation.

\section{ACCORDI REGIONALI DI INTEGRAZIONE, UNA ALTERNATIVA DI SVILUPPO}

\section{Riassunto}

Dalla seconda guerra mondiale i rapporti giuridici tra i diversi paesi che compongono la comunità internazionale ha subito grandi trasformazioni derivate, principalmente dal nuovo ordine di potere che scaturiva dalla conflagrazione universale che si è verificato tra il 1938 e il 1945. Questa nuova organizzazione basata su rapporti d'affa- 
ri che rendono necessaria l'integrazione tra i diversi Stati, al fine di raggiungere livelli di sviluppo sostenibile in un mondo sempre più specializzato nella catena di produzione. Questa situazione porterà a che, nelle sottoregioni diverse del pianeta, sgorgano Accordi Regionali d'integrazione i qualli cercherà di unire gli sforzi degli Stati, al fine di raggiungere una capacità economica globale che consente ulteriormente lo sviluppo. Questa tendenza non esce dell'America Latina, nonostante le difficoltà che sorgono a causa della rottura ideologica dei diversi gruppi che compongono la regione e gli interessi particolari delle corporazioni politici, industriale e commerciale che vede, l'atomizzazione degli Stati, un particolare modo di conseguire i propri interessi, situazione che ha portato al fallimento i tentativi di realizzare una vera integrazione regionale.

Parole chiave: Accordo Regionale d'integrazione, ideologia, integrazione, regionalizzazione.

\section{OS ACORDOS REGIONAIS DE INTEGRAÇÃO, UMA ALTERNATIVA DE DESENVOLVIMENTO}

\section{Resumo}

A partir da segunda guerra mundial os relacionamentos jurídicos entre os diferentes países que integram a comunidade internacional sofreram grandes mudanças derivadas, principalmente, da nova ordem de poder que surgiu da revolução universal que se deu entre os anos 1938 e 1945. Esta nova organização parte das relações comerciais que fazem necessária a integração entre os diferentes Estados, a fim de atingir níveis de desenvolvimento sustentável em um mundo cada vez mais especializado na cadeia de produção. Esta situação vai chegar a que, nas diferentes sub-regiões do planeta surjam Acordos Regionais de Integração que vão tentar unir os esforços dos Estados, em procura de atingir uma capacidade econômica global que providencie um maior desenvolvimento. A esta tendência não foge a América Latina apesar das dificuldades que se apresentam por causa ao rachamento ideológico dos diferentes grupos que compõem a região e aos interesses particulares dos grêmios políticos, industriais e comerciais que 
vem, na atomização dos Estados, uma maneira particular de atingir seus próprios interesses, tudo isso tem levado ao fracasso as tentativas para atingir a verdadeira integração regional.

Palavras-chave: Acordo Regional de Integração, alfandegárias, ideologia, regionalização. 


\section{INTRODUCCIÓN}

Las tendencias actuales de la globalización y de la implementación del neoliberalismo como nueva corriente ideológica económica ha llevado a que algunos países aglutinen sus intereses con la conformación de grupos económicos a través de los cuales puedan protegerlos, en un mundo cada vez más competitivo y marcado por un consumismo acelerado que ha conllevado la ruptura de los mercados tradicionales y de las formas clásicas de establecer las negociaciones de carácter comercial.

Bajo esta premisa surgen diversos tratados como los Tratados de Libre Comercio (TLC) que son acuerdos bilaterales entre dos países, sean o no circunvecinos, y los Acuerdos Regionales de Integración (ARI), entendidos como acuerdos multilaterales entre países que deben ser circunvecinos y que acuerdan unas estrategias comerciales, políticas y jurídicas que les permiten competir en igualdad de condiciones con otros bloques regionales.

Este tipo de acuerdos han permitido, al menos en regiones como el continente europeo y en Norteamérica, que se inicien procesos de integración que han trascendido, incluso, la esfera de lo meramente comercial para entrar a configurar, de manera lenta pero estable, la construcción de un derecho que, poco a poco, trasciende todas las esferas de aplicación y que propende por una unificación del mundo jurídico conforme a los intereses particulares de cada región, como una premisa para la construcción de un derecho de carácter universal.

Desde la perspectiva propuesta cada vez es mayor la voluntad de lograr Acuerdos Regionales de Integración en el ámbito mundial, pero cuando se observa el panorama específico de América Latina se encuentra que si bien han surgido algunos acuerdos (G3, PA, MCCA y MERCOSUR, entre otros) no existe uno que aglutine la totalidad de los países que componen la región.

Ello puede deberse a diferentes causas que habrá que analizar desde una perspectiva holística, toda vez que cada una de ellas y todas en conjunto pueden ser fundamento para lograr un acercamiento de los diferentes Estados en procura de una auténtica integración.

Entre estas causas se deben resaltar la herencia colonial recibida de España, los obstáculos geográficos que dificultan la unidad territorial, la falta de voluntad política de los gobernantes por circunstancias ideológicas, los intereses propios de los grupos industriales locales, los nacionalismos radicales que dividen a los pueblos latinoamericanos, la falta de una identi- 
dad nacional latinoamericana y los niveles de desigualdad comercial entre los distintos países que componen la región. Además, no debe olvidarse que:

el regionalismo es un asunto complejo, que depende en gran manera de ciertas características particulares de casos específicos. No hay reglas que sean aplicables a las políticas sobre Acuerdos Regionales de Integración que sean a la vez universales y operacionales. Las primeras son de tal amplitud que pierden capacidad operativa, mientras que las operacionales son demasiado específicas para ser universales (Schiff y Winters, 2004).

Para entender la trascendencia que tienen los procesos de integración es necesario observar que experiencias como la Comunidad Económica Europea (CEE) han demostrado que una vez que se logran unificar los criterios comerciales se hace necesaria la creación de figuras jurídicas de aplicación general para todos los integrantes del ARI, en áreas como el derecho comercial, laboral, civil, penal e incluso administrativo, constitucional e internacional puesto que se presentan, obligatoriamente, relaciones de derecho privado y público, dada la naturaleza propia de la actividad contractual. Si América Latina lograra trascender las problemáticas internas concretando acuerdos de cobertura regional se empezaría a caminar en torno a la creación de un derecho unificado latinoamericano, con lo cual desaparecería, o al menos se mitigaría, la fragmentación tradicional de los países que componen la región.

Bajo estas premisas la investigación que se adelanta, denominada "Acuerdos Regionales de Integración. Derecho latinoamericano, presente y futuro", de la cual se presenta hoy un primer informe, pretende, en primer lugar, hacer un análisis de los expectativas y realidades que los ARI han generado en el ámbito de la internacionalización a nivel mundial para, posteriormente, en una segunda fase, entrar a estudiar las circunstancias específicas que rodean el accionar político, económico y cultural de América Latina, como una subregión que, desde su misma conformación histórica, se ha comportado de manera errática cuando se trata de unificar los criterios de los distintos grupos de poder que ejercen el dominio sobre los territorios que la componen; por ello se parte desde un interrogante que deberá resolverse para alcanzar la pretensión de una integración de la región, tratando de identificar cuáles han sido las causas que no han permitido en América Latina la creación de un ARI, como un paso previo a la constitución de un derecho 
unificado para toda la región, interrogante que deberá ser resuelto gracias al desarrollo de unos objetivos propios como son la determinación de las principales características de un ARI, definiendo las ventajas y desventajas que surgen a raíz de la firma y puesta en ejecución de un acuerdo de integración económica; igualmente, deberán estudiarse los diferentes acuerdos de integración económica que se han suscrito a partir de la Segunda Guerra Mundial analizando, de manera particular, los suscritos en América Latina.

Otro paso previo que deberá conducir al objetivo general que se plantea la investigación es la caracterización de las diferencias históricas, económicas, políticas y culturales que se presentan en las diversas subregiones de América Latina, insumo que servirá para, en una etapa final, realizar un estudio de derecho comparado, constitucional, legal y jurisprudencial entre los principales países de América Latina, en búsqueda de unos elementos comunes que concluyan de manera definitiva esta etapa de desunión histórica y que permita que América Latina pueda enrumbarse hacia el destino que la espera como potencia mundial, una vez que las ideologías y los intereses de grupo sean desbordados por la realidad evidente del desarrollo potencial que puede alcanzar la región.

\section{Metodología}

Si bien es innegable que tratándose de una investigación en la cual están inmersos unos resultados de orden económico, que hacen referencia, principalmente, al desarrollo en términos de producción, de producto interno bruto de los Estados y de las posibilidades de un mejoramiento económico no sólo de los países que conforman la comunidad internacional sino de las comunidades que los habitan, no puede hablarse que la presente sea un investigación cuantitativa en sentido estricto, puesto que más allá de estas magnitudes de orden económico se perfila la intencionalidad de demostrar las causas que han impedido que América Latina alcance unos niveles de integración que posibiliten su desarrollo local y global.

Es por ello que la metodología utilizada se ha venido enfocando más desde el orden cualitativo puesto que, en el fondo lo que se persigue, a través de la presentación de magnitudes económicas, es la comprensión de fenómenos de orden político y social y no un esquema frío de unos porcentajes de desenvolvimiento económico que, si bien son evidencia del éxito o del fracaso de las acciones que se desarrollan, no dan cuenta de los factores so- 
ciales, políticos y culturales que han fraccionado históricamente a América Latina.

Desde la perspectiva en la cual se ha proyectado la investigación se establece un primer momento de orden exploratorio, dado que se trata de contextualizar los esfuerzos que se han realizado en torno a la integración de los Estados, en su búsqueda, más allá de los resultados objetivos que los acuerdos suscritos hayan dejado como muestra tangible frente a la actividad económica de los países signatarios de dichos acuerdos.

En cuanto a las fuentes estas han sido, en esta primera etapa, de orden documental, lo cual no es óbice para que en el desarrollo posterior de la investigación se deba acudir a la recolección de datos tanto de orden cuantitativo como cualitativo, mediante la aplicación de encuestas y entrevistas. Hasta el momento la información se ha tomado consultando libros, revistas especializadas, e información publicada en diferentes periódicos, haciendo énfasis en aquellos que están centrados en temas económicos, al igual que se ha acudido a información encontrada a través de las páginas web que abordan la temática propuesta. Es importante anotar que se ha tomado información no sólo de los ARI que han operado durante los últimos decenios en el contexto mundial, de manera general, y en América Latina, de manera particular, sino que se ha acudido a la búsqueda de datos históricos que permitan dar cuenta de las problemáticas sociales, económicas y políticas del continente latinoamericano.

Los datos recabados en esta primera parte han sido analizados aplicando formatos de análisis documental que han sido estructurados por los integrantes del grupo, para los datos que se recojan a futuro se utilizarán las técnicas tradicionales de la estadística para los datos de orden cuantitativo y de la categorización de variables para los datos cualitativos, según el esquema planteado por Strauss y Corbin (2002).

\section{DESARROLLO}

\section{Apuntes históricos}

Si bien, cuando se habla de procesos de integración no puede desconocerse que en la antigüedad se dieron unos primeros conatos del dominio de una potencia que, sin acercarse siquiera al concepto moderno de integración, se constituyeron en Estados dominantes que marcaron de manera 
significativa la economía y el mundo político y militar de las regiones bajo su influencia:

El nacimiento de la primera potencia se fija en la vejez, más precisamente en el siglo VII a. C, con la creación de Roma, siete siglos más tarde se convertiría en la primera potencia mundial, también conocida como el Imperio Romano. La influencia de la edad antigua en Roma es indudable, desde hace miles de años, su cultura, la política y la economía han influido en varias civilizaciones.

A mediados del siglo XIV, otros países se transforman en las potencias mundiales y esto se debe a la Ilustración de Francia, España y Portugal, debido al descubrimiento de nuevas tierras en el nuevo mundo, a través de la navegación.

Más tarde, los laureles de la revolución industrial impulsan a Inglaterra para que se convierta en la potencia más importante, esto debido a que mantiene su poder hasta finales de la Segunda Guerra Mundial (Dalmagro y López, 2011).

A pesar de estos antecedentes históricos, para los efectos de la investigación propuesta se partirá de un hito que hizo ver a los Estados la necesidad de iniciar procesos de unificación, bajo criterios no sólo económicos sino políticos, jurídicos, sociales, culturales e incluso militares:

[...] El Tratado de Westfalia que fue la conclusión de la guerra de los treinta años (siglo XVII) y dio soberanía a pequeños estados de Europa Central es, ante todo, el fin de la Contrarreforma de Fernando II y su intención de recuperar el poder universal de la religión católica. Westfalia es el fin del proyecto del Imperio Universal (fundamentado en lo religioso) y el reconocimiento de los estados soberanos (inicialmente europeos) como los protagonistas del nuevo orden mundial de la época (Cardona, 2010, p. 13).

A partir de allí se presenta un enfrentamiento en la concepción que, de la internacionalización, tienen los Estados; por un lado los Estados Nación propenden por la creación de un Estado universal, soportado en la concepción religiosa y la supremacía de los Estados europeos, y por otro la concepción liberal que cimenta sus ideas en la soberanía y autodeterminación de los Estados.

Por su parte Kant (2005) concibe las relaciones internacionales desde los conceptos de colaboración, integración y negociación entre los Estados, 
teoría que tendrá mucha incidencia en las concepciones modernas de las relaciones internacionales y dará pie a la conformación de la Sociedad de las Naciones y la terminación de la Primera Guerra Mundial.

\begin{abstract}
Contrario al Liberalismo Idealista, el Realismo — surgido después de la Segunda Guerra Mundial e inspirado en el Leviathan de Hobbes-, concibe las relaciones entre estados como de conflicto, no de solidaridad. Una evidencia empírica de la mirada realista de las Relaciones Internacionales puede ser la constitución, al interior del Sistema de Naciones Unidas, de un Consejo de Seguridad liderado por las potencias vencedoras de la Segunda Guerra Mundial. Los protagonistas de la Guerra Fría habrían de sentarse en este escenario para resolver sus permanentes conatos de guerra. Posterior a la Guerra Fría, también ha servido para legitimar acciones de guerra contra naciones que no hacen parte de este selecto grupo de potencia.

En este orden de ideas, el surgimiento de propuestas sobre un gobierno mundial o Federal también refleja el enfrentamiento entre corrientes. Mientras los liberales ven en la concertación racional un espacio para evitar el conflicto, realistas como Morgenthau se apoyan en una concepción pesimista de la naturaleza humana para colocar la lucha por el poder como el eje de las relaciones internacionales. O sea, las negociaciones entre Estados y los acuerdos derivados de estas no son el resultado de la solidaridad internacional sino de la resolución de conflictos en la búsqueda del poder (Cardona, 2010, pp. 14, 15).
\end{abstract}

Lo importante de estas tendencias es que van a conformar una conciencia de la necesidad de unificar algunos aspectos que han sido exclusivos de la soberanía de los países, dado que en las relaciones internacionales, cuando se observa la realidad económica mundial, con miras a enfrentar los retos que plantea el siglo XXI, se hace evidente que existe una marcada propensión a la integración de bloques económicos y políticos de carácter subregional. Este fenómeno puede observarse al analizar la Comunidad Económica Europea, la Alianza de los denominados Tigres Asiáticos y los esfuerzos de integración económica realizados por los países de Norteamérica (NAFTA).

Todos ellos nacidos a mediados del siglo xx ya que antes de la Segunda Guerra Mundial el sistema económico imperante era el denominado librecambismo, teoría económica que puede definirse como el sistema económico que propugna por la libre circulación de las mercancías entre 
Estados y la eliminación de los aranceles; sistema que fue impulsado principalmente por Inglaterra y que permite el libre intercambio de productos entre países procurando que la intervención de las autoridades económicas sea limitada al máximo al fin de sacar el mayor provecho a las condiciones de producción propias de un determinado país. Este sistema está apoyado en dos conceptos que van a ser básicos para su desarrollo: la división del trabajo y el patrón oro, y va a llevar a que los países con mejores índices de producción alcancen ventajas comparativas en el intercambio de productos.

Durante finales del siglo XIX y principios del siglo XX el librecambio fue el sistema económico imperante en los países de influencia occidental pero, a partir de la depresión del año 1929, como consecuencia del colapso económico derivado principalmente como secuela de la Primera Guerra Mundial, los Estados tuvieron que adoptar medidas proteccionistas a fin de resguardar su producción interna.

Esta nueva situación lleva a una variación en los niveles de poder económico y los Estados Unidos comienzan a perfilarse como la primera potencia del mundo, lo que permite la implementación de acuerdos bilaterales con otros países con los cuales se llega a un consenso para establecer una reducción de aranceles bajo los principios de la reciprocidad y de la nación más favorecida, principio que implica que si un Estado, que ha firmado un acuerdo con otro, concede una ventaja comparativa a un tercero, esta ventaja se hace extensiva al inicialmente firmante.

Este sistema de negociación bilateral va a ser muy frecuente en el período entre guerras pero, al terminar la Segunda Guerra Mundial, el mundo va a sufrir cambios drásticos a los cuales no va a ser ajena la actividad comercial entre Estados.

En primer lugar debe tenerse en cuenta que la destrucción sufrida por los países europeos va a ser de grandes proporciones, razón por la cual, para su reconstrucción, van a recibir, inicialmente, ayudas económicas por parte de Estados Unidos, fundamentalmente gracias al denominado Plan Marsha11, a través del cual la potencia norteamericana inyecta fondos a los países del continente europeo a cambio de ventajas comerciales que terminan siendo una amenaza para la independencia económica de los países de la región.

Esta situación lleva a la necesidad de la creación de organismos financieros que permitan que los Estados puedan acceder a recursos y a condiciones favorables para el desarrollo de su actividad comercial, entre ellos, el Banco Internacional de Reconstrucción y Fomento, el Fondo Monetario Internacional y la Organización Mundial del Comercio. Posteriormente, se 
establece el Acuerdo General sobre Tarifas Aduaneras y Comercio (GATT), con lo cual se busca pasar de acuerdos bilaterales a tratados multilaterales sin que con ello se cierre la posibilidad que dos o más países puedan aliarse para crear Zonas de Libre Comercio y Uniones Aduaneras, siempre y cuando no afecten a otros países con los cuales se hayan firmado acuerdos multinacionales.

Los países europeos van a tomar la iniciativa en la conformación de organizaciones multilaterales, en busca de unos intereses comunes, toda vez que era necesario un intercambio comercial de algunos productos que permitiesen cerrar un poco la dependencia frente a los Estados Unidos, razón por la cual se iniciaron los primeros pasos en torno a la Unión Europea.

\begin{abstract}
[...] dos franceses, Jean Monnet y Robert Schumann, creían que Francia y Alemania serían capaces de superar los mutuos recelos y antagonismos si existieran los suficientes incentivos económicos que estimularan la cooperación. En el mes de Mayo de 1950, Schumann propuso la creación de una autoridad común y única para regular la industria del carbón y del acero en Alemania y Francia, aunque la oferta estaba abierta a otros países europeos con intereses en estos sectores productivos. La idea fue muy bien acogida y el 18 de abril de 1951 tuvo lugar la firma del Tratado de París, siendo sus signatarios la República Federal Alemana, Bélgica, Holanda, Luxemburgo, Italia y Francia. Este tratado de París daría lugar, el 10 de agosto de 1952 a la Comunidad Europea del Carbón y del Acero (CECA).

El objetivo económico del tratado era establecer un mercado común del carbón y del acero, es decir, suprimir aranceles, subvenciones nacionales o medidas discriminatorias para asegurar así la libre competencia en este sector, obtener precios más baratos y un abastecimiento sin interrupción (Hernández y Díaz, 2010).
\end{abstract}

La CECA se constituye en un antecedente para los procesos de integración regional que llevarán, en un primer momento, a la creación de otras organizaciones de integración europea como la Comunidad Europea de la Energía Atómica (EURATOM) y la CEE, con lo cual el concepto de Acuerdos Regionales de Integración toma forma, siendo así que, a partir de allí y en todos los continentes, se van a conformar grupos regionales y subregionales encaminados a lograr una integración que, si bien en un principio es básicamente comercial, terminará incorporando entre sus objetivos la vinculación de otros aspectos jurídicos y sociales que son necesarios para alcan- 
zar una verdadera integración, tales como los aspectos laborales, penales, de libertad de fronteras e, incluso, constitucionales, con lo cual se amplía la aplicación del derecho entre los países partes de los tratados que crean dichos grupos de integración.

Como puede observarse la integración europea parte, fundamentalmente, de necesidades de carácter económico, tal como se desprende de los objetivos expuestos en el preámbulo del Tratado de Roma, mediante el cual los países signatarios deciden, definitivamente, la creación de un Comunidad Económica Europea. Dichos objetivos se pueden sintetizar de la siguiente manera: sentar las bases de una unión estrecha entre los pueblos europeos, asegurar, a través de una acción conjunta, el progreso económico y social mediante la eliminación de las barreras que dividen a los diferentes países, mejorar las condiciones de vida y de trabajo de los pueblos, la garantía de un desarrollo económico sostenible, el intercambio comercial estable y la competencia leal, el reforzamiento de las economías locales mediante la reducción de las diferencias entre las regiones más y menos favorecidas, la paulatina supresión de las restricciones a los intercambios internacionales y la consolidación de la defensa de la paz y la libertad (Tratado de Roma, 1957).

A partir de esta experiencia europea comienzan a surgir, en América Latina y en otras regiones del mundo, varios acuerdos de integración regional los cuales, dado que aún se conservaba una mentalidad proteccionista y debido a los intereses nacionalistas, no alcanzaron de manera plena los objetivos propuestos.

La supervivencia y el aparente éxito de la CEE dio lugar, en los años sesenta, a una racha de regionalismo entre los países en desarrollo. Esta fue motivada en gran medida por la creencia, entre quienes abogaban por la sustitución de las importaciones, de que la protección era necesaria para la industrialización - y, por ende, para la prosperidad- y de que esta política resultaría menos costosa si se extendía a un área económica más amplia. Los ARI (Acuerdos Regionales de Integración) fueron, por lo general, muy proteccionistas e intervencionistas, en el sentido de que trataron de determinar por vía administrativa qué industrias establecer y en dónde ubicarlas. Esto implicaba numerosos controles y restricciones a la actividad económica y, por consiguiente, produjo resultados económicos bastante modestos. De otro lado, la ejecución fue a menudo lenta, en parte debido a los desacuerdos respecto a dónde se deberían situar las industrias. Hacia finales de 
los setenta la ineficacia de estos ARI era clara. Se hacía evidente que ninguno de estos acuerdos había contribuido decididamente al desarrollo; algunos de ellos habían fracasado y las tensiones provocadas por la crisis de la deuda dejaron agonizantes a gran parte de los pocos acuerdos que sobrevivieron (Schiff y Winters, 2004, p. 5).

Pronto será superada esta mentalidad restringida y limitante la cual sufre un cambio radical. Los diferentes bloques regionales comprenden, de manera acertada, que mediante una unión encaminada a un intercambio comercial libre de trabas arancelarias y de toda índole se puede lograr un mayor desarrollo e industrialización.

Se van a conformar, entonces, los Acuerdos de Integración Regional, unos más exitosos que otros, en prácticamente todas las subregiones del planeta. Como un ejemplo de ello, en el continente americano se concertarán el Acuerdo de Libre Comercio entre Estados Unidos y Canadá (NAFTA) y al cual se unirá posteriormente México; el Mercado Común Centroamericano (MCCA); el Grupo de los Tres (G3), integrado por México, Colombia y Venezuela; la Comunidad Andina de Naciones (CAN) y el Mercado Común del Sur (MERCOSUR); en el continente asiático la Asociación de Naciones del Sudoeste Asiático (AFTA) y, en África, el Mercado Común del Este y Sur de África (COMESA), todos ellos acordados bajo el criterio de un mejoramiento de la economía de los países miembros a través de un esfuerzo encaminado a fortalecer la calidad y el volumen de la oferta de bienes y servicios.

\section{Conceptualizaciones generales}

No obstante, el concepto de Acuerdo de Integración Regional no es un criterio unificado puesto que puede hablarse de diferentes tipos de acuerdos como, por ejemplo, las uniones aduaneras, que consiste en el acuerdo de dos o más países para eliminar, entre ellos, los aranceles, al tiempo que mantienen uno en común frente a los países que no están vinculados a la unión; las zonas de libre comercio, que son acuerdos con los cuales los países miembros no sólo eliminan los aranceles entre ellos sino que permiten el libre comercio con los otros Estados Parte, pudiendo mantener los aranceles propios de cada país frente a los demás Estados no signatarios del acuerdo; existen además tratados bilaterales y multilaterales, obedeciendo al número de países miembros, los acuerdos sectoriales, cuando sólo se aplican a de- 
terminados sectores de la producción o globales cuando cobijan a todos los sectores productivos; se clasifican, así mismo, en naturales o abiertos cuando no hay restricciones entre los Estados Parte; estratégicos, cuando existen políticas propias para los países participantes que no se hacen extensivas a los países no miembros del acuerdo; homogéneos, en el evento en el cual los países signatarios avanzan a un mismo ritmo en el cumplimiento de los compromisos y heterogéneos, cuando el desarrollo es establecido de acuerdo a las capacidades propias de cada Estado.

En este orden de ideas es necesario destacar que al hablar de Acuerdos Regionales de Integración este término lleva a la comprensión de otros que, significando cada uno su propia conceptualización semántica, están encaminados a explicar diferentes tópicos que confluyen de manera general en el concepto macro de integración regional. Entre estas conceptualizaciones, conocidas básicamente como Acuerdos Preferenciales de Comercio, cabe destacar lo siguiente:

Acuerdo preferencial: es la forma más simple de integración económica en la cual se otorgan determinadas ventajas de manera recíproca entre los firmantes.

Área de libre comercio: el área de libre comercio es un acuerdo entre países que busca el desmonte de las barreras al comercio interior con el fin de dar libertad al movimiento de mercancías en la zona.

Unión aduanera: la unión aduanera es el acuerdo entre países que supone, además de la eliminación de barreras comerciales entre los países miembros, una política arancelaria común frente al resto de países.

Mercado común: es el área económica en la cual hay libre movimiento de mercancías y factores de producción, como consecuencia de la eliminación total de los controles aduaneros internos y el levantamiento delas barreras no arancelarias existentes

Unión económica: es el nivel máximo de integración, donde se establece una moneda única y políticas macroeconómicas sectoriales y sociales comunes. Lo que trae consigo una pérdida de la soberanía nacional para los países miembros de esta.

Integración económica plena: la integración económica plena se da cuando se contemplan dentro de un acuerdo los siguientes requisitos: libre comercio de mercancías, arancel externo común, libre movilidad de factores política económica armonizada y política económica unificada (Carbonell, García, Llerena, Maury, Ortiz, et al., 2012). 


\section{El caso particular de América Latina}

Frente al caso particular de Latinoamérica es necesario acotar que se ha presentado una multiplicidad de acuerdos tanto multi como bilaterales que han permitido que, de manera incipiente, se empiece a configurar una identidad latinoamericana.

En este sentido debe entenderse, para una mejor comprensión, que el término multilateral hace referencia a acuerdos celebrados entre varios países que no son, todos ellos, pertenecientes a la misma región, es decir, no son en su totalidad países vecinos; tal es el caso del denominado G3 compuesto por Colombia, Venezuela y México.

El término bilateral, por su parte, hace relación a acuerdos suscritos entre dos países que pueden ser o no vecinos, como puede observarse con los Tratados de Libre Comercio suscritos por los Estados Unidos con México, su vecino natural, con Colombia, un socio necesario en los intereses norteamericanos en la región y con Chile, país ubicado en el otro extremo geográfico.

Finalmente, deberá entenderse como Acuerdo Subregional de Integración a los tratados comerciales firmados por varios países que son vecinos entre sí, tal como acontece con el Mercado Común del Sur, acuerdo suscrito por Brasil, Argentina, Uruguay y Paraguay, países todos ellos limítrofes y que por ende, es de suponer, poseen unas características y unas necesidades comunes.

Al tomar el término Acuerdo Regional de Integración, concepto al cual está encaminado en esencia el trabajo investigativo que se desarrolla, deberá entenderse, entonces, una propuesta de unidad inicialmente comercial pero que deberá encaminarse a la unificación de otros aspectos tales como lo laboral, lo penal e incluso lo constitucional, conformada por todos los países que integran el bloque continental conocido como América Latina y no aquellos acuerdos que están limitados a unos determinados países con prescindencia de la totalidad de los países que conforman el área latinoamericana.

Los proyectos de integración de América Latina han sido múltiples puesto que algunos dirigentes tienen conciencia que estructurando un Acuerdo Regional de Integración latinoamericano podrían darse las bases para alcanzar niveles de desarrollo que permitan solucionar los problemas endémicos de la población, tales como educación, desnutrición, falta de agua potable $\mathrm{y}$, en general, falta de recursos para lograr niveles de dignidad de todos los latinoamericanos. 
Es de observar que de lograrse la creación de un organismo verdaderamente representativo no sólo el mundo económico latinoamericano vería fortalecidos sus criterios para intentar una unificación industrial y comercial sino que la comunidad política y académica alcanzaría, a través de estudios de estas características, elementos suficientes para una mayor comprensión de la problemática regional, con lo cual se sentarían las bases para propuestas encaminadas al mejoramiento del nivel de vida de todos los latinoamericanos, lo cual exigiría de los gobernantes una voluntad encaminada no a los intereses particulares de cada país o de cada grupo en el poder sino de un verdadero desarrollo de la región.

Desde esta perspectiva de una unión que permita el desarrollo global de la región se han suscrito algunos acuerdos encaminados a conseguir una verdadera integración regional que, a pesar de los conflictos ideológicos, económicos, sociales y culturales que ha suscitado, pretende sentar las bases para alcanzar, en un mediano plazo, unos verdaderos niveles de integración regional.

[...] en 1960 se funda una organización de amplio alcance que fue la Asociación Latinoamericana de Libre Comercio (ALALC). Esta asociación, a través de su sucesora, la Asociación Latinoamericana de Integración (ALADI), concretada en 1980, se encuentra activa al día de hoy. En 1975, asimismo, se crea el Sistema Económico Latinoamericano y del Caribe (SELA).

En cuanto a las instituciones subregionales y luego de una serie de antecedentes previos, en 1951 se crea la Organización de Estados Centroamericanos (ODECA), seguida en los años sesenta por el Secretariado Centroamericano para la Integración Económica (SIECA). Posteriormente, el Sistema de Integración Centroamericana (SICA) se constituye en la organización económica, cultural y política de los Estados de Centroamérica desde su inicio en 1993. En los años que van de 1965 a 1972 funciona la Asociación Caribeña de Libre Comercio (CARIFTA), que fue sucedida por la actualmente vigente Comunidad del Caribe (CARICOM), establecida a partir de 1973. En el Cono Sur se funda en el año 1969 el Pacto Andino, que se relanzaría con un nuevo nombre, Comunidad Andina de Naciones (CAN), a partir de 1996. A partir de un acuerdo de Cooperación Económica Argentina-Brasil de 1985 se sientan los antecedentes de lo que sería el Mercosur, establecido formalmente a partir de 1991. En el año 2001 se da lugar a la creación de la Alianza Bolivariana para los Pueblos de Nuestra América (ALBA). En el año 2000 comienzan las tratativas para una 
unificación de los procesos de integración en el Cono Sur, CAN y Mercosur, que darán lugar a la Unasur en 2008, cuyo antecedente es la Declaración de Cuzco (2004), que establece la creación de la Unión de Naciones de América del Sur (CELAC, 2010).

No obstante las buenas intenciones y los esfuerzos realizados en busca de iniciar de manera firme los procesos de integración de la región han sido múltiples los obstáculos que se han presentado y que no han permitido que se alcancen unos verdaderos niveles de cooperación abierta entre los diferentes países que han suscrito los acuerdos, lo cual ha representado, más allá de las buenas intenciones, un fracaso en la permanencia y eficacia de dichos proyectos.

Para entender la magnitud de este fracaso es necesario partir de la innegable realidad que confirma que las economías latinoamericanas están signadas por profundas desigualdades que se dan no sólo por los diversos desarrollos industriales y económicos, endémicos en la región, sino por profundas diferencias culturales, políticas e ideológicas que no han permitido, hasta el momento, la creación de verdaderos proyectos regionales de integración económica.

La integración regional sudamericana enfrenta una serie de obstáculos, entre ellos los geográficos, estructurales y políticos. La dura realidad geográfica del continente con las barreras de la Cordillera de los Andes y la Cuenca Amazónica dificulta la conectividad y comunicaciones entre este y oeste, norte y sur. La infraestructura de conectividad es muy costosa y el transporte es limitado y lento. Cualquier proyecto de integración debe superar las grandes limitaciones infraestructurales de conectividad, de forma social y ambientalmente sostenible, en el continente.

Aún si estos obstáculos geográficos pudieran ser superados, la región enfrenta otros obstáculos políticos y estructurales. Si bien parte de la región hereda un acervo ideológico y político poderoso del pensamiento de grandes luchadores por la independencia como Bolívar o Martí, que apuntan a una Patria Grande y una región unida, la realidad política de un continente fragmentado se mantiene como uno de los principales obstáculos para la integración (Ticehurst, 2008).

Desde la misma época de la colonización española estas diferencias se hicieron evidentes dado que algunas regiones se desarrollaron rápidamen- 
te, en tanto se dejaron por fuera de los parámetros "civilizadores" a grandes zonas del continente, tal como sucedió con la zona central de América Latina que prácticamente fue olvidada por los europeos. Esta desigualdad entre los polos de desarrollo y las dificultades geográficas que presenta la región determinaron una heterogeneidad cultural y económica que aún sobrevive. Cada subregión fue asumiendo su propio modo de vida y generando una incipiente industrialización y unas posibilidades comerciales propias de su contexto, pero no se alcanzó un grado de desarrollo generalizado que permitiera pensar en una unificación de políticas que diesen mayores posibilidades de progreso a la región como una pantotalidad.

Esta situación continúa a pesar de que hoy, gracias a los desarrollos técnicos y tecnológicos alcanzados y a la experimentación de algunos grupos subregionales, sería válido pensar en la posibilidad de una integración económica que abarcase a todos los países que componen el área ya que ello podría generar un surgimiento económico que convirtiera a la región en un bloque fuerte frente a los demás bloques regionales, puesto que es pertinente observar que un acuerdo regional de integración latinoamericano permitiría potenciar la región, que cuenta con grandes recursos sin explotar y con algunos polos de desarrollo importantes como México y Brasil, entre otros, y que, gracias a los esfuerzos unidos de todos los países que componen el área, se convertiría en un mercado interno con un recurso humano potencial de más de seiscientos millones de habitantes y que cuenta con cerca del $20 \%$ del agua potable del mundo y múltiples recursos naturales que permitirían que, mediante una concertación multilateral, se unificasen los criterios industriales y comerciales para una adecuada explotación de dichos recursos, encaminando la producción y la comercialización hacia el mercado interno en condiciones controladas.

Sin embargo, estas potencialidades no son tenidas en cuenta por los líderes que dirigen los destinos de las naciones, poniendo de presente que la falta de integración se debe no sólo a las barreras geográficas y culturales sino a los intereses de los grupos económicos, sujetos a una dependencia endémica que ha sido causa del poco desarrollo político y social de la región:

La primera razón del fracaso no está en las economías de escala, en las técnicas y procedimientos para la rebaja arancelaria o en cualquier otro aspecto de técnica económica y tampoco está en la economía "pura", si es que esta existe de algún modo.

Esa razón se encuentra en esa zona donde la economía se amalgama con la política, la sociología, la historia y la cultura para explicar 
el fracaso de la burguesía industrializante que para la CEPAL era el principal actor social que debía hacer cambios estructurales internos imprescindibles (reforma agraria para quebrar el latifundio y la acción del regresivo binomio latifundio-minifundio, redistribución del ingreso, sin lo cual el mercado interno seguiría siendo estrecho), disponerse a resistir con firmeza la penetración y dominio de las transnacionales en defensa de sus mercados nacionales y de su mercado regional y por tanto, disponerse a enfrentar a los gobiernos de Estados Unidos, sin lo cual es impensable alguna política de desarrollo autónoma en esta región.

Como balance regional, y sin olvidar que cada historia nacional es específica y nunca exacta a otras, la burguesía industrializante soñada por CEPAL fracasó en su papel como estrella del reparto. Demostró ser más transnacionalizada que nacional y por lo general, aceptó la hegemonía norteamericana y el actuar como administradores de la dependencia y empleados de alto nivel de filiales de transnacionales, antes que ser los burgueses "nacionales", plantados en defensa de sus mercados, empresas y proyectos propios (Ticehurst, 2008).

Dependencia esta que se puso en evidencia con la autorización concedida por Colombia a Estados Unidos cuando, en desarrollo del Plan Colombia, autorizó el establecimiento de bases militares norteamericanas en territorio colombiano, lo cual fue interpretado por algunos países de la región como un clara injerencia en los asuntos locales, creando así una situación tensa que resquebrajó, en su momento, la orientación integradora de la UNASUR.

El día 30 de octubre de 2009 - y como una continuación natural del fracasado Plan Colombia- los gobiernos de Colombia y Estados Unidos suscribieron un nuevo acuerdo que permite la presencia de efectivos y civiles estadounidenses en bases militares colombianas en función de reforzar la lucha contra el narcotráfico y la guerrilla. A la firma de este Tratado precedió un tenso escenario entre los meses de agosto y octubre, el cual puso a prueba la solidez de UNASUR. Con la intención de encontrar respaldo a esta iniciativa entre sus pares, el presidente Uribe inició una gira diplomática por siete países sudamericanos entre los días 4 y 6 de agosto con miras a la reunión que se realizaría en Quito el día 10 de agosto. Uribe tuvo éxito sólo en Perú, Chile y Paraguay, de cuyos presidentes obtuvo respaldo sobre la base del respeto a las decisiones soberanas de cada país. El resto de los paí- 
ses se mostró reticente a aceptar el acuerdo, siendo Brasil uno de los principales opositores al objetar abiertamente el tratado (Bywaters, Cristóbal. Rodríguez, Isabel y otros, 2010)

Ello ha llevado a que a pesar de estos esfuerzos mancomunados y de las buenas intenciones expresadas por los gobernantes de turno no haya sido posible que estos intentos de integración funcionen de una manera tal que se dé un beneficio generalizado para los países que conforman la región, puesto que los intereses de los grupos locales ha llevado a que cada vez sea más marcada la inequidad frente a amplios sectores sociales, sobre todo de aquellos que componen los países menos favorecidos, lo cual ha permitido que cada día haya una mayor injusticia social y que no se alcancen los objetivos puestos en el papel.

[...] Para los pocos que han sido capaces de realizar alguna transformación productiva de relieve, las políticas públicas han sido parcas en resultados, cuando no regresivas en términos de segregación social. O sea, no parece ser posible emprender políticas sociales compensatorias capaces de contrarrestar los efectos de políticas económicas neoliberales que suponen entregar al mercado la instancia decisoria sobre prioridades para la aplicación de recursos financieros escasos e inestables. El pragmatismo del Poder y del Dinero ha oscurecido las esperanzas de tantos que lucharon durante años por la transición democrática como instrumento de negociación nacional de los intereses de nuestros pueblos. La sumisión de la mayoría de nuestros gobiernos (quizás por no haberse completado aún la democracia en muchos países o por no haberse profundizado lo suficiente en otros) ha mermado la capacidad de ampliación de una alianza verdadera entre los países de América Latina (Conceiao y Gomes, 2015).

Como un rechazo a esta situación comienza a surgir, lenta y medrosamente, una nueva conciencia política que, lamentablemente, no ha sido acogida de manera general por todos los países de la región pero que sí empieza a perfilar un grupo fuerte de rechazo a las políticas de Estados Unidos, sembrando las semillas de lo que podría llegar a ser una política que propenda por la integración latinoamericana desde criterios netamente regionales, como una contraposición a las políticas neoliberales que imperan actualmente en el mundo económico global, dominado por los países altamente desarrollados. 
[...] en pocos años emergió un nuevo liderazgo político que reemplazó al que durante varias décadas había conducido los destinos de la región: Luiz Inácio Lula da Silva en Brasil, Tabaré Vázquez en Uruguay, Hugo Chávez en Venezuela, Rafael Correa en Ecuador, Evo Morales en Bolivia y Néstor Kirchner en Argentina. Estos nuevos gobiernos, aun cuando han cuestionado el neoliberalismo y las posturas hegemónicas de Estados Unidos, no han construido todavía un proyecto económico claramente alternativo. Pareciera existir cierta perplejidad para enfrentar los desafíos del desarrollo en la actual fase de globalización y ciertas dudas acerca de cómo ganar posiciones competitivas, especialmente frente a China y la India. Pero lo más paradójico es que estos gobiernos no manifiestan una clara voluntad integracionista. Lula lideró el rechazo al ALCA y cuestionó la posición de Estados Unidos, que insistía en la apertura en servicios e inversiones junto a la protección de la propiedad intelectual y al mismo tiempo rechazaba una discusión amplia sobre los mecanismos antidumping y los subsidios agrícolas. Pero, pese a ello, Brasil no ha sido capaz, o no ha querido, ejercer un liderazgo que avance definitivamente en la integración regional. Por su parte, el gobierno de Kirchner concentró sus esfuerzos en resolver los problemas internos heredados del periodo anterior y dedicó poco espacio a la política internacional y regional. Ecuador y Bolivia se encuentran en una situación similar a la de Argentina, con el agravante de que se han embarcado en asambleas constituyentes. Venezuela, finalmente, despliega un activismo incesante para acumular fuerza interna, al tiempo que intenta afirmar posiciones de liderazgo en Sudamérica, con una retórica que ha generado varios conflictos en la región (Pizarro, 2008, p. 30).

Derrotero este que muestra, de manera fehaciente, que existe una tendencia en la actualidad encaminada no sólo, como ha sido tradicional, a un acercamiento de los países latinoamericanos con las grandes potencias, Estados Unidos y Europa, y más modernamente los países asiáticos y los grandes emporios industriales de oriente sino que, de manera paulatina, se toma conciencia de la necesidad de establecer Acuerdos Regionales de Integración entre los países que componen la región usufructuando de esta manera las ventajas comparativas que traen consigo la unión en aspectos tan fundamentales como la integración económica, política y cultural, por lo cual se hace necesario profundizar en el estudio de las problemáticas internas latinoamericanas a fin de superar los escollos de una división generalizada entre grupos ideológicamente distintos encaminados a la satisfacción 
de sus propios intereses, aunque ello vaya en desmedro de una verdadera integración de la región.

\section{CONCLUSIONES}

A través de la historia han existido Estados que bajo el ejercicio de la fuerza o mediante una adecuada diplomacia se han convertido en el epicentro de la cultura y el desarrollo, unificando a diversos pueblos bajo su dominio político y, fundamentalmente, económico, logrando de esta manera potenciar las ventajas comparativas que da el poseer un mayor territorio y una mejor estructura en los aspectos industriales y comerciales.

Esta tendencia, que en la antigüedad permitió la conformación de los grandes imperios, ha sufrido unas profundas transformaciones en los tiempos modernos, en los cuales ya no es el poder de la guerra el que unifica a las naciones sino las actividades comerciales y económicas que se han constituido en el eje fundamental de las relaciones internacionales.

Bajo este criterio se deben observar con mucho cuidado los acercamientos entre Estados, en el orden comercial, jurídico, político e, incluso, social y cultural, porque de ellos se están derivando grandes consecuencias, al menos económicas, que están transformando las relaciones entre los Estados y creando nuevas estructuras de poder internacional que van sepultando, poco a poco, el concepto tradicional del poder.

Esta nueva dinámica internacional ha traído consigo una verdadera transformación de la actividad que se despliega tanto del sector privado como desde el sector público y ha generado una nueva especialización en la división internacional del trabajo y en las estructuras macro que definen la actividad estatal, llevando, incluso, a que se esté pensando, en algunos bloques subregionales, en la integración no sólo comercial sino laboral, penal e incluso constitucional del derecho, con lo cual se evitan los conflictos derivados de la aplicación exegética de unos ordenamientos jurídicos propios de cada Estado que todavía están cimentados en el prosaico concepto del Estado Nación que surgió como consecuencia de las evoluciones políticas propias del siglo XVIII.

Se hace necesario que estas corrientes de integración que se han convertido en las banderas que defienden los países más industrializados sean absorbidas como parte integral de su propia existencia por los países que componen las diversas subregiones del planeta en las cuales se hayan ubicados los países mal denominados tercermundistas, es decir, en vías de 
desarrollo, a fin de alcanzar unas metas de producción local que no serán posibles si cada nación sigue adelantando, de manera aislada, sus procesos, convirtiendo así a los pueblos en simples conglomerados humanos manipulados por pequeños grupos económicos que, en su miopía histórica, no alcanzan a comprender las ventajas que trae consigo el trabajar con el otro en la búsqueda de un bien común.

América Latina no puede ser ajena a estos procesos de modernización y de globalización, máxime cuando se tienen unas mismas raíces y unos mismos antecedentes históricos que siempre han estado presentes a pesar de las ambiciones de pequeños grupos políticos y económicos que desunen los pueblos, en aras de la consecución de sus intereses personales.

La integración que se viene gestando desde la década de los años cincuenta del siglo $\mathrm{xx}$ ha generado procesos que han representado grandes fracasos frente al sueño de la unión latinoamericana pero que también han aportado unas experiencias valiosas que permiten, hoy en día, que por encima de los intereses de grupos locales y de líderes populistas pueda soñarse con una unificación de los intereses propios de América Latina, como una región de un potencial único, en un planeta que se agota cada día a un ritmo acelerado.

Corresponde a los líderes de las naciones americanas, a sus dirigentes, industriales, economistas, clase académica y cultural y a todos los estamentos que componen la región, abandonar los chovinismos que han fragmentado Latinoamérica convirtiéndola en lo que Halperín (1998) denomina, de manera acertada, las treinta jóvenes Américas, un continente fraccionado por los intereses particulares de los líderes locales que impone sus propias mezquindades por encima de los intereses de una región que merece un mejor futuro y una gloria mayor.

Se hace, por lo tanto, necesario ahondar en la investigación de los procesos de integración de los países que conforman el área latinoamericana pues, es a través de ellos que se podrán alcanzar los niveles de desarrollo que se necesitan para superar la pobreza, el analfabetismo, las condiciones sociales y culturales endémicas de la región y, sobre todo, la miseria humana que cubre el corazón de sus dirigentes que, sumidos en su propio beneficio, se olvidan que el ser humano es algo más que un instrumento temporal para el alcance de las metas que les fija su propia ambición. 


\section{REFERENCIAS BIBLIOGRÁFICAS}

Bywaters, C., Rodríguez, I. et al. (2010). "Unasur y la integración latinoamericana: propuesta de un nuevo modelo del regionalismo postliberal". Revista Encrucijada Americana, año 3, 1, 16, 17.

Carbonell, M. A., García, G., Llerena, B., Maury, A., Ortiz, M., et al. (2012). "Niveles de integración económica de los acuerdos comerciales en Colombia". Recuperado de http://es.slideshare.net/BeatrizLlerena/niveles-de-integracin-econmica-de-los-acuerdos-comerciales

Cardona, G. (2010). La integración regional en Europa y América Latina: reflexiones desde la óptica de las teorías de las relaciones internacionales y de la economía. Medellín, Colombia: CEIPA.

CELAC Comunidad de Estados Latinoamericanos y Caribeños (2010). Fortalecimiento y profundización de los procesos de integración de las economías de América Latina y el Caribe. México D. F.: México.

Conceiao, M. y Gomes, G. (2015). "La CEPAL y la integración económica de América Latina”. Recuperado de http://archivo.cepal.org/pdfs/ revistaCepal/Sp/0NE213228.pdf

Dalmagro, A. y López, G. E. (2011). "Las potencias emergentes y su impacto en la integración latinoamericana. Primer Congreso Internacional de la Red de Integración Latinoamericana 2011". Recuperado de http:// www.uncu.edu.ar/relacionesinternacionales/upload/redilaeje23.pdf

Halperín, T. (1998). Historia contemporánea de América Latina. Madrid, España: Alianza Editorial.

Hernández, F. y Díaz, J. R. (2010). "La Unión Europea: del Tratado de Roma al Tratado de Lisboa". Recuperado de http://hdl.handle. net/10553/3322 les Tecnos.

Kant, I. (2005). Sobre la paz perpetua. Salamanca, España: Editoria-

Pizarro, R. (2008). "El difícil camino de la integración regional". Revista Nueva Sociedad, 214, 30.

Resico, M. F. (2011). Introducción a la economía social del mercado. Bogotá, Colombia: Instituto Konrad Adenauer Stiftung.

Schiff, M. y Winters, L. A. (2004). Integración regional y desarrollo. Bogotá, Colombia: Alfaomega - Banco Mundial.

Strauss, A. y Corbin, J. (2002). Bases de la investigación cualitativa, técnicas y procedimientos para desarrollar la teoría fundamentada. Medellín, Colombia: Editorial Universidad de Antioquia. 
Ticehurst, S. (2008). “¿Nuevos horizontes para la integración americana?". Revista Umbrales, 17.

Tratado de Roma (1957). Mediante el cual se crea la Comunidad Económica Europea. Roma, Italia. 
